\title{
ON THE FOUNDATIONS OF QUASIGROUPS
}

\author{
BY \\ SHERMAN K. STEIN(1)
}

1. Introduction. Quasigroups have two fundamental properties which groups in general lack. First, a quasigroup may be homogeneous in the sense that its group of automorphisms may be transitive. The only group with this property has just one element. Second, a quasigroup has a rich outer symmetry (or duality) in the sense that to each quasigroup are associated six conjugate quasigroups (defined in $\S 3$ ), one for each element of the symmetric group on three letters. In general, only the transpose of a group is again a group.

Any constraint satisfied by a quasigroup yields a conjugate constraint satisfied by a conjugate quasigroup. The theory of conjugate constraints and conjugate quasigroups is developed and illustrated in $\$ \S 2-8$. Of special interest are constraints which are invariant, that is, constraints which are equivalent to their six conjugates. The constraints of associativity and commutativity are not invariant. In fact, as is pointed out in $\$ 5$, one of the conjugates of associativity is the identity $a b \cdot a c=b c$. Thus, the theory of groups is equivalent to the theory of quasigroups satisfying the identity $a b \cdot a c=b c$. Considered from this point of view, the theory of groups may seem artificial. In any case, the existence of the tremendous theory of groups suggests that quasigroups satisfying other constraints than associativity and commutativity might merit consideration.

For example, the constraint of mediality $a b \cdot c d=a c \cdot b d$, implied by the conjunction of associativity and commutativity, is invariant and has been investigated. $i$ shall be considered in detail in $\$ \$ 11-14$. The constraint of left-distributivity $a \cdot b c=a b \cdot a c$, which is intertwined with mediality, has received less attention. It shall be treated in $\$ \S 9,10$. The only left-distributive group is the trivial group. This serves to illustrate another of the attractions of quasigroups - they may satisfy identities which usually conflict with associativity. Also, in groups it is usually obvious whether a constraint is or is not satisfied; therefore, a theory of constraints has not been developed for groups.

In $\S \S 15,16$ the relation of orthogonality to an algebra is studied. In particular, a new technique for constructing orthogonal quasigroups is presented which may produce a counterexample to Euler's conjecture. Conjugates of

Received by the (ditors June 26, 1956.

(1) Part of the work on this paper was done while the author held a Summer Faculty Fellowship from the University of California. The translation of portions of Mituhisa [25], by Makoto Yaguchi, was sponsored by the Department of Mathematics at the University of California at Davis. 
various constraints are listed in $\S \S 17,18$. In $\$ 19$ conjugation is applied to varieties.

Historical remarks, comments, and suggestions for future work are collected in $\$ \$ 20,21$ under the title of Commentary.

\section{Conjugation}

2. Algebras. Let $X$ be a set and $n \geqq 1$ be an integer. An algebra on $X$ is a function $f: X^{n} \rightarrow X$ which is defined for all elements of $X^{n}$ and is single-valued. In $X^{n+1}, f$ defines a subset $G$ which shall be called the graph of $f$ :

$$
G=\left\{\left(x_{1}, \cdots, x_{n}, f\left(x_{1}, \cdots, x_{n}\right)\right) \mid x_{i} \in X\right\} .
$$

If $\pi_{i}: X^{p} \rightarrow X$ denotes projection parallel to the $i$ th axis of $X^{p}$ then a subset $H \subset X^{n+1}$ is the graph of an algebra if and only if

$$
\pi_{n+1} \mid H: H \rightarrow X^{n}
$$

is an equivalence (i.e., a one-one onto function).

Let $S_{k}$ denote the symmetric group on the first $k$ integers. Each $\sigma \in S_{k}$ induces an equivalence on $X^{k}$, again denoted $\sigma$, defined by

$$
\sigma\left(\left(x_{1}, \cdots, x_{k}\right)\right)=\left(x_{\sigma(1)}, \cdots, x_{\sigma(k)}\right) .
$$

If $G$ is the graph of an algebra $f$ and $\sigma \in S_{n+1}$ with $\sigma(n+1)=n+1$ then $\sigma G$ is also the graph of an algebra which shall be denoted $\sigma f$. For example, if $n=2$ and $\sigma=(12)$ then $f$ is a binary algebra and $\sigma f$ is its transpose.

\section{Polyadic algebra.}

Definition 3.1. If the graph $G$ of $f$ satisfies the more stringent demand

$$
\pi_{i} \mid G: G \rightarrow X^{n} \quad 1 \leq i \leq n+1
$$

are equivalence, $f$ (or $G$ ) is a polyadic algebra.

For example, if $n=1, f: X \rightarrow X$ is an equivalence. For $n=2, f: X^{2} \rightarrow X$ is a quasigroup. Explicitly, the fact that $\pi_{1}$ is an equivalence asserts the existence and uniqueness of the solution to the equation $x a=b$; the same property of $\pi_{2}$ implies the same for the equation $a x=b$ (the notation $x y$ is the standard abbreviation for $f(x, y))$.

Definition 3.2. For each $\sigma \in S_{n+1}$ and polyadic algebra $G \subset X^{n+1}, \sigma G$ is a polyadic algebra and shall be called a conjugate of $G$ (or of $f$ ) and may also be denoted $\sigma f$.

The notion of conjugacy clearly includes that of transposition. There may be $n+1$ ! nonisomorphic algebras conjugate to an $n$-ary algebra.

4. Conjugate quasigroups. The case $n=2$ of polyadic algebras shall be the only one considered in what follows. If $f(a, b)=c$ then $(a, b, c) \in G$. If $\sigma \in S_{3}, \sigma(a, b, c) \in \sigma G$. For example, if $\sigma=(13)$ then $(c, b, a) \in \sigma G$. This fact may be written $(\sigma f)(c, b)=a$ or, with an ambiguity that shall be removed by the context, simply $c b=a$. The following table records the six quasigroup conjugate to a given quasigroup $f$. 


\begin{tabular}{|c|c|c|c|c|c|c|}
\hline$\sigma$ & $I$ & (12) & (13) & (23) & (123) & (132) \\
\hline equation & $f(a, b)=c$ & $(\sigma f)(b, a)=c$ & $\sigma f(c, b)=a$ & $\sigma f(a, c)=b$ & $\sigma f(b, c)=a$ & $\sigma f(c, a)=b$ \\
\hline abbreviation & $a b=c$ & $b a=c$ & $c b=a$ & $a c=b$ & $b c=a$ & $c a=b$ \\
\hline
\end{tabular}

Definition 4.2. An associative quasigroup is a group. A quasigroup with a two-sided unit is a loop. A quasigroup in which the square of each element is itself is an idempotent quasigroup.

THEOREM 4.3. The quasigroups conjugate to a group $f$ may be defined in terms of $f$ and the inverse operation in $f$ as follows:

\begin{tabular}{|c|c|c|c|c|c|c|}
\hline$\sigma$ & $I$ & (12) & (13) & (23) & (123) & (132) \\
\hline$\sigma f(a, b)$ & $f(a, b)$ & $f(b, a)$ & $f\left(a, b^{-1}\right)$ & $f\left(a^{-1}, b\right)$ & $f\left(b^{-1}, a\right)$ & $f\left(b, a^{-1}\right)$ \\
\hline abbreviation & $a b$ & $b a$ & $a b^{-1}$ & $a^{-1} b$ & $b^{-1} a$ & $b a^{-1}$ \\
\hline
\end{tabular}

where the last row refers to the law $f$.

Proof. Consider the case $\sigma=(132)$. Let $(\sigma f)(a, b)=c$. Then $(a, b, c) \in \sigma G$, where $G$ is the graph of $f$. Solve the equation $(a, b, c)=\sigma(x, y, z)$ for $x, y, z$. That is, $(a, b, c)=(y, z, x)$. Thus, $y=a, z=b, x=c$. Hence, $(c, a, b) \in G$; equivalently, $b=c a$, or $c=b a^{-1}$. The five remaining cases are similar.

It should be observed that in general a conjugate of a group (loop) is not a group (loop), except for the cases $\sigma=I$, the group (loop) itself, and $\sigma=(12)$, its transpose, (and also a special case considered in $\$ 7$ ).

\section{Conjugate constraints.}

DEFINITION 5.1. A constraint on $f$ is a collection of assertions of the type; if $t_{1}, \cdots, t_{k} \in G$ then $t \in G$, where $t$ depends on $t_{1}, \cdots, t_{k}$.

For example, the identity $a \cdot b c=a b \cdot c$ on $f$ may be translated into the constraint:

$$
(b, c, x),(a, x, y),(a, b, u) \in G \text { imply }(u, c, y) \in G .
$$

Definition 5.2. A constraint on $f$ induces an equivalent constraint on $\sigma f$ by the demand:

$$
\text { if } \sigma t_{1} \cdots, \sigma t_{k} \in \sigma G \text { then } \sigma t \in \sigma G,
$$

where $\sigma t$ depends on $\sigma t_{1}, \cdots, \sigma t_{k}$. Such an equivalent constraint is a conjugate constraint.

For example, if $\sigma=(23)$ the constraint of associativity for $f$ becomes for of the constraint:

$$
(b, x, c),(a, y, x),(a, u, b) \in \sigma G \text { imply }(u, y, c) \in \sigma G .
$$

This may be written in the functional notation of the algebra $\sigma f$ as

$$
c=b x, x=a y, b=a u \text { imply } u y=c .
$$


Or equivalently $c=a u \cdot a y$ implies $c=u y$. This is simply the identity

$$
a u \cdot a y=u y .
$$

Thus, the identity 5.3 is a conjugate of the identity of associativity; it of course can be written $a b \cdot a c=b c$.

THEOREM 5.4. The conjugates of the constraint of associativity are the identities of the following table:

\begin{tabular}{|c|c|c|c|c|c|c|}
\hline$\sigma$ & $I$ & (12) & (13) & (23) & (123) & (132) \\
\hline identi & $a \cdot b c=a b \cdot c$ & $a \cdot b c=a b \cdot c$ & $b a \cdot c a=b c$ & $a b \cdot a c=b c$ & $b a \cdot c a=b c$ & $a b \cdot a c=$ \\
\hline
\end{tabular}

Proof. The case $\sigma=(23)$ is discussed above. The remaining cases are similar.

COROLlaRy 5.6. The theory of groups is equivalent to the theory of quasigroups satisfying the identity $a b \cdot a c=b c$.

As will be shown in $\$ 18$ the conjugate of an identity, while it is of course a constraint, is not necessarily expressible as an identity.

6. Symbolic logic of constraints. An examination of the details involved in determining the constraints conjugate to associativity in the previous section shows that all the computations could be carried out in a completely formal manner, independently of the existence of algebras or quasigroups. The computations involve only certain properties of the sign " =", functions, and substitutions. It is the object of this section to define "constraint" and "conjugate of a constraint" formally and also more precisely than in the preceding section.

Let $\mathcal{F}$ be an elementary functional calculus with no individual constants, individual variables $a, b, c, \cdots, x, y, z, \cdots$, one function symbol of degree 2 , written ".", and one relation symbol of degree $2, "=$ ". The other symbols of $\mathcal{F}$ are $\supset, F,[],,(),, \wedge$ standing respectively for "implies", "false", "left and right bracket", "left and right parentheses", and "all".

Definition 6.1. A string is a finite sequence of symbols from the above list.

Definition 6.2. A string $\alpha$ is a term if there is a sequence $\alpha_{1}, \cdots, \alpha_{n}=\alpha$ such that for all $i, 1 \leqq i \leqq n$, either

(1) $\alpha_{i}$ is an individual variable or (2) $\alpha_{i}$ is $\left(\alpha_{j} \cdot \alpha_{k}\right)$, with $j, k<i$.

The following lemma is usually tacitly assumed when interpreting a term in a model for $\mathcal{F}$.

Lemma 6.3. If $A, B, A^{\prime}, B^{\prime}$ are terms and $(A \cdot B)$ is $\left(A^{\prime} \cdot B^{\prime}\right)$ then $A$ is $A^{\prime}$ and $B$ is $B^{\prime}$.

Proof. It suffices to show that $A$ is $A^{\prime}$. By symmetry it can be assumed that $A$ is an initial section of $A^{\prime}$. However, the number of left parentheses 
of a proper initial section of any term exceeds the number of right parentheses of this section by at least one. Thus, $A$ must coincide with $A^{\prime}$.

Definition 6.4. A string $A$ is a well-formed formula if there is a sequence $A_{1}, \cdots, A_{n}(=A)$ such that for all $i, 1 \leqq i \leqq n$, either (1) $A_{i}$ is $F,(2) A_{i}$ is $\alpha=\beta$ where $\alpha, \beta$ are terms, (3) $A_{i}$ is $\left[A_{j} \supset A_{k}\right], j, k<i$ or (4) $A_{i}$ is $(\bigwedge b) A_{j}$, $j<i$ where $b$ is an individual variable.

Within the well-formed formulas are singled out certain ones, called theorems, by specifying axioms and the notion of proof. The axioms, for example, would assert that "=" is an equivalence relation and "." a quasigroup. Moreover, the logical operations $\sim$ (not), $\wedge$ (and), $\vee$ (or), $\equiv$ (equivalent), can be defined in terms of the symbols already introduced; for example, $\sim A$ is an abbreviation for $[A \supset F]$.

Definition 6.5. A constraint is a well-formed formula of $\mathcal{F}$.

Definition 6.6. A constraint of the form $\alpha=\beta$, where $\alpha$ and $\beta$ are terms, is an identity.

Henceforth, the symbols " $\left({ }^{\prime},{ }^{\prime}\right)$ ", and ".", will be deleted if ambiguity does not arise; $(a \cdot b)$ shall be written $a b$.

Definition 6.7. An atomic constraint is a constraint such that each expression contained in it of the form $\alpha=\beta$ involves at most one multiplication (that is, is of the form $a b=c$ or $a=b$ ).

As the method of the following examples shows, any constraint is equivalent to an atomic constraint.

EXAmple. An atomic constraint equivalent to the identity $a b=b a$ is $[a b=x \supset b a=x]$.

EXAmple. Atomic formulas equivalent to the identity $a \cdot b c=a b \cdot c$ are

$$
[(b c=x) \wedge(a x=y) \wedge(a b=z) \wedge(z c=w) \supset(y=w)]
$$

and also

$$
[(b c=x) \wedge(a x=y) \wedge(a b=z) \supset(z c=y)] .
$$

Definition 6.10. If $\sigma \in S_{3}$ and $\gamma$ is a constraint then the conjugate constraint $\sigma \gamma$ is obtained by replacing $\gamma$ by an equivalent atomic constraint and then replacing each expression of the type $x_{1} x_{2}=x_{3}$ by $x_{\sigma(1)} x_{\sigma(2)}=x_{\sigma(3)}$.

ExAmple. Let $\gamma$ be $a \cdot b c=a b \cdot c$ and $\sigma=(23)$. An atomic form for $\gamma$ is, say, (6.9). Therefore $\sigma \gamma$ is

$$
[b x=c \wedge a y=x \wedge a z=b \supset z y=c] .
$$

The logic of $\mathcal{F}$ shows that this is equivalent to $a b \cdot a c=b c$.

Definition 6.11. The subgroup of $S_{3}$ consisting of those $\sigma$ such that $\sigma \gamma \equiv \gamma$ is the group belonging to $\gamma$. If the group of $\gamma$ is $S_{3}$ then $\gamma$ is invariant.

THEOREM 6.12. If the constraint $\gamma$ implies $\delta$ then $\sigma \gamma$ implies $\sigma \delta$.

Proof. Application of $\sigma$ to the steps of the proof that $\gamma$ implies $\delta$ yields a proof that $\sigma \gamma$ implies $\sigma \delta$. (For example, see Corollary 11.8). 
COROLLARY 6.13. If the constraint $\gamma$ implies the invariant constraint $\delta$ then $\sigma \gamma$ implies $\delta$.

Corollary 6.14. If the invariant constraint $\gamma$ implies the constraint $\delta$ then $\gamma$ implies $\sigma \delta$.

If $f$ is an algebra on $X$ and $\sigma$ is a term with $k$ distinct individual variables then $\sigma$ induces a function $g: X^{k} \rightarrow X$, by interpreting ". " as " $f$ " and the individual variables in $\sigma$ as running through $X$. That $g$ is well-defined is a consequence of Lemma 6.3. Not only terms, but, in a similar manner, constraints can be interpreted in an algebra.

Definition 6.15. The algebra $f$ is a model for the constraint $\gamma$ if the interpretation of $\gamma$ in $f$ is valid; $f$ is also said to satisfy $\gamma$.

Definition 6.16. If $\gamma$ is a constraint, $J(\gamma)$ consists of the integers which are orders of quasigroup models of $\gamma$.

$J(\gamma)$ is clearly closed under multiplication if $\gamma$ is an identity.

THEOREM 6.17. If the quasigroup $Q$ satisfies the constraint $\gamma$ and $\sigma \in S_{3}$ then $\sigma Q$ satisfies the constraint $\sigma \gamma$.

7. Illustrations of the technique of conjugate constraints. The following theorems are illustrations of the preceding observations on constraints.

Theorem 7.1. A quasigroup $Q$ satisfies the identity $a b \cdot b c=a c$ if and only if it is a group which is a power of the group of order two.

Proof. To show $a b \cdot c=a \cdot b c$ solve the equations $a=x y, b=y z, c=z t$. Then

$$
\begin{aligned}
& a b \cdot c=(x y \cdot y z) z t=x z \cdot z t=x t, \\
& a \cdot b c=x y(y z \cdot z t)=x y \cdot y t=x t .
\end{aligned}
$$

Thus $Q$ is a group. Let $e$ be its unit element. Then $e b \cdot b e=e^{2}$. Then $b^{2}=e$ for all $b$, proving the theorem.

Corollary 7.2. $J(a b \cdot b c=a c)=\left\{2^{i}, i=0,1,2, \cdots\right\}$.

COROLlary 7.3. If all the conjugates of a group $G$ are groups, then $G$ is a power of the group of order two (and conversely).

Proof. Since $a e=a, \sigma G$, for $\sigma=(23)$, satisfies the equation $a^{2}=e$. Since $\sigma G$ is a group it must be a power of the group of order two, and hence satisfy $a b \cdot b c=a c$. This identity is invariant (see $\$ 17$ ); by Theorem $7.1, G$ is itself a power of the group of order two. The converse follows from the invariance of $a b \cdot b c=a c$.

Theorem 7.4. A loop $Q$ satisfies the identity $a b \cdot a c=d b \cdot d c$ only if it is a group which is a power of the group of order two.

Proof. Since $d$ may equal $e$, which is a left unit, $Q$ satisfies $a b \cdot a c=b c$, 
which is conjugate by $\sigma=(23)$ to associativity. $\sigma Q$ is therefore a group. Also, since $e$ is a right unit $x e=x$ in $Q$. Thus, $x x=e$ in $\sigma Q$. Hence $\sigma Q$ is a power of the group of order two. Therefore, $Q$ is also.

Lemma 7.5 (Suschkewitsch [3]). In a quasigroup $Q$ the following two constraints are equivalent:

$$
\begin{aligned}
B: a b \cdot c & =a \cdot b d \text { implies } a^{\prime} b^{\prime} \cdot c=a^{\prime} \cdot b^{\prime} d, \\
B^{\prime}: a b & =c d \text { implies } a \cdot b x=c \cdot d x .
\end{aligned}
$$

Proof. Assume $Q$ satisfies $B$ and let $a b=c d$. Then $a \cdot b x=c \cdot d y$ for some $y^{\cdot}$ Also $a \cdot b x=a b \cdot z=c d \cdot z$. But the equations $a b \cdot z=a \cdot b x, c d \cdot z=c \cdot d y$, together with $B$, imply $y=x$, hence $B^{\prime}$.

Assume $Q$ satisfies $B^{\prime}$. Choose $e$ so $b e=b$. For given $a, b, d$ find $c$ so $a b=c d$. By $B^{\prime} a \cdot b e=c \cdot d e$. Thus $e$ is a right unit. Now let $a b=q=q e$. By $B^{\prime}$

$$
a \cdot b c=q \cdot e c=a b \cdot e c .
$$

Since $e c$ is independent of $a$ and $b, \dot{B}$ follows.

COROLLARY 7.6. In a quasigroup the following two constraints are equivalent:

$$
\begin{gathered}
A: a b=c d \text { implies } x a \cdot x b=y c \cdot y d, \\
A^{\prime}: a b \cdot a c=d b \cdot d c .
\end{gathered}
$$

Proof. For $\sigma=(23), A^{\prime}=\sigma B^{\prime}$ (Table 18.1). Also, $A=\sigma B$. The corollary is a consequence of Lemma 7.5 and Theorem 6.12.

8. Conjugates of commutativity. A second well-known constraint is $a b=b a$. Straightforward computation yields the following table for the constraints conjugate to it.

\begin{tabular}{c|c|c|c|c|c|c}
$\sigma$ & $I$ & $(12)$ & $(13)$ & $(23)$ & $(123)$ & $(132)$ \\
\hline Constraint & $a b=b a$ & $a b=b a$ & $b \cdot b a=a$ & $a b \cdot b=a$ & $b \cdot b a=a$ & $a b \cdot b=a$ \\
\hline
\end{tabular}

Definition 8.2. An algebra satisfying all the identities conjugate to commutativity is totally symmetric.

Theorem 8.3. A quasigroup $f$ with graph $G$ is totally symmetric if and only if $\sigma G=G$ (or $\sigma f=f)$ for all $\sigma \in S_{3}$.

Proof. The commutativity of $f$ is equivalent to $\sigma G=G$, for $\sigma=(12)$. That $f$ satisfies $a b \cdot b=a$ is equivalent to $\sigma G=G$, for $\sigma=(13)$. Since $S_{3}$ is generated by any two distinct transpositions the theorem is proved.

Corollary 8.4. The quasigroup $f$ is totally symmetric if and only if $a b=c$ implies $b a=c$ and $c b=a$.

THEOREM 8.5. There exist totally symmetric idempotent quasigroups only for orders $n \equiv 1,3(\bmod 6)[47]$. 
THEOREM 8.6. A totally symmetric algebra is a (totally symmetric) quasigroup.

Proof. Since $a b \cdot b=a$, the equation $x b=a$ has at least one solution. Assume that $x b=y b$. Then $x b \cdot b=y b \cdot b$, which implies $x=y$. Similarly the constraint $b \cdot b a=a$ implies the equation $b x=a$ has precisely one solution.

Corollary 8.7. An algebra $f$ satisfying the constraints $a b \cdot b=a=b \cdot b a$ is $a$ commutative quasigroup (hence totally symmetric quasigroup).

Proof. In view of the preceding theorem and its proof it is sufficient to show that $f$ is totally symmetric, and hence commutative. Since $\sigma f=f$ for $\sigma=(23)$ and $\sigma=(13)$ and any two transpositions generate $S_{3}, f$ is totally symmetric.

\section{Distributivity}

9. Self-distributivity.

Definition 9.1. The algebra $f$ is left- (right-) distributive if $f$ satisfies $a \cdot b c=a b \cdot a c(b c \cdot a=b a \cdot c a)$.

The conjugates of left-distributivity are listed in the following table:

\begin{tabular}{|c|c|c|c|c|c|c|}
\hline$\sigma$ & $I$ & (12) & (13) & (23) & (123) & $(132)$ \\
\hline Constraint & $a \cdot b c=a b \cdot a c$ & $b c \cdot a=b a \cdot c a$ & $\begin{array}{c}a b=c d \text { implies } \\
a c \cdot b d=a b(=c d)\end{array}$ & $a \cdot b c=a b \cdot a c$ & $b c \cdot a=b a \cdot c a$ & $\begin{array}{l}a b \cdot c d \text { implies } \\
a c \cdot b d=a b=c d\end{array}$ \\
\hline
\end{tabular}

Left-distributivity is an example of an identity possessing a conjugate constraint which is not an identity.

Theorem 9.3. A left-distributive quasigroup is idempotent.

Proof. Set $a=b=c$. Then $a \cdot a^{2}=a^{2} \cdot a^{2}$. Cancellation implies $a=a^{2}$.

Corollary 9.4. A quasigroup satisfying a constraint conjugate to leftdistributivity is idempotent.

Proof. Theorem 9.3 and Corollary 6.13.

THEOREM 9.5. The center ${ }^{2}$ ) of a left-distributive quasigroup $Q$ is either void or all of $Q$.

Proof. Let $b_{0} c=c b_{0} \cdot$ for all $c \in Q$. Then, $a \cdot\left(b_{0} c\right)=a \cdot\left(c b_{0}\right)$ or $a b_{0} \cdot a c=a c \cdot a b_{0}$ for all $a, c \in Q$. Thus, $a b_{0}$ is in the center of $Q$ for all $a$. Thus $Q$ is abelian.

THEOREM 9.6. An abelian left-distributive quasigroup $Q$ satisfies all constraints conjugate to left-distributivity.

Proof. That $Q$ is right-distributive is clear. To show that $A B=C D$ implies

(2) The center of an algebra $A$ is the set of elements which commute with all the elements of $A$. 
$A C \cdot B D=A B$, solve the equations $x=A, x y=C, x z=D$ successively for $x, y, z$. Then, since $A B=C D$ and $Q$ is left-distributive, $y z=B$. Then

$$
\begin{aligned}
A C \cdot B C & =(x \cdot x y)(y z \cdot x z)=(x \cdot x y)(y x \cdot z)=(x \cdot x y)(z \cdot y x) \\
& =(x \cdot x y)(z \cdot x y)=y z \cdot x y=x \cdot z y=x \cdot y z=A B,
\end{aligned}
$$

proving the theorem.

THEOREM 9.7. $Q$ is an abelian left-distributive quasigroup if and only if it satisfies the identity $\gamma, a b \cdot c a=a \cdot b c$.

Proof. Set $a=b=c$ in $\gamma$. Then, $a^{2} \cdot a^{2}=a \cdot a^{2}$, so $Q$ is idempotent. Next set $a=b$ in $\gamma$. Then $b b \cdot c b=b \cdot b c$. Cancellation yields $b c=c b$. The remainder of the proof is equally direct.

Lemma 9.8. Let $Q$ be a finite idempotent quasigroup of order $n$, and $x$ an element of $Q$. Introduce on the nondiagonal elements, $t$, of $G$, containing $x$ as one of its three coordinates a symmetric relation $R$ defined by: $t R t^{\prime}$ if and only if there is $i, 1 \leqq i \leqq 3$, so that $\pi_{i} t$ is the transpose of $\pi_{i} t^{\prime}$. Let $\phi(x)$ equal the number of equivalence classes of the minimal equivalence relation generated by $R$. If $Z=\sum_{x} \phi(x)$ then

$$
Z \equiv \frac{n(n-1)}{2}(\bmod 2) .
$$

Proof. The (topological) proof of this theorem is to be found in [46].

THEOREM 9.9. There exist left-distributive quasigroups of order $n$ only for $n \equiv 0,1,3(\bmod 4)$.

Proof. Existence will be established first. On the Galois field $G F\left(2^{k}\right), k \geq 2$, introduce the law of composition $x \circ y=a x+\beta y$ with $\alpha$ and $\beta$ fixed nonzero elements satisfying the equation $\alpha+\beta=1$. A simple computation shows that the algebra $f$ defined by $f(x, y)=x \circ y$ is a left-distributive quasigroup.

On the cyclic group of odd order $m$ introduce a law of composition $x \circ y=x^{p} y^{q}$, with $(p, m)=1=(q, m)$ and $p+q \equiv 1(\bmod m)$. Again a simple computation shows that $x \circ y$ defines a left-distributive quasigroup.

Now $n \equiv 0,1,3(\bmod 4)$ is equivalent to $n=2^{k} \cdot m, k \neq 1, m$ odd. The orders of the quasigroups constructed above and their direct products sweep out all such $2^{k} \cdot m$.

Nonexistence will next be considered. A left-distributive quasigroup has a transitive group of automorphisms. In fact, the left translations constitute a transitive set of automorphisms. It is not difficult to see that if, in an idempotent quasigroup, there is an automorphism $\alpha$ with $\alpha(x)=x^{\prime}$ then $\phi(x)$ $=\phi\left(x^{\prime}\right)$. Thus, if the group of automorphisms is transitive $Z \equiv 0(\bmod n)$. If $n$ is even $Z$ is therefore even. But, if $n=2 m, m$ odd, $Z$ is odd, since $Z \equiv(n)(n-1) / 2(\bmod 2)$. This contradiction establishes the theorem. 
Definition 9.10. The algebra constructed from the $G F\left(2^{k}\right)$ by the multiplication $x \circ y=a x+\beta y$, where $\alpha, \beta$ are fixed in $G F\left(2^{k}\right)$ shall be denoted $A\left(G F\left(2^{k}\right), \alpha, \beta\right)$. The algebra constructed on the cyclic abelian group $G(n)$, of order $n$, by the multiplication $x \circ y=x^{p} \cdot y^{q}$, where $p, q \cdot \in J$ shall be denoted $A(G(n), p, a)$.

COROLlary 9.11. The group of automorphisms of a quasigroup of order $n \equiv 2(\bmod 4)$, containing at least one idempotent element, is not transitive.

Proof. A quasigroup with at least one idempotent element and possessing a transitive group of automorphisms is idempotent. The method of the preceding proof therefore applies.

Theorem 9.12. Let $Q$ be a left-distributive quasigroup and $a, b \in Q$. The equivalence $\phi: Q \rightarrow Q$ defined by $a x=b \phi(x)$ is an automorphism.

Proof. Like that of Theorem 9.13, to follow.

TheOREM 9.13. Let $Q$ be a left- and right-distributive quasigroup and $a \in Q$ be a fixed element. The equivalence $\phi: Q \rightarrow Q$ defined by $a x=\phi(x)$ a is an (inner) automorphism.

Proof. Let $a x=\phi(x) a$ and $a y=\phi(y) a$. Then $a(x y)=a x \cdot a y=\phi(x) a \cdot \phi(y) \cdot a$ $=\phi(x) \phi(y) \cdot a$. Thus $\phi(x y)=\phi(x) \phi(y)$.

THEOREM 9.14. Let $Q$ be a left- and right-distributive quasigroup and $a, b \in Q$. The equivalence $\phi: Q \rightarrow Q$ defined by $a x=\phi(x) \cdot b$ is an automorphism.

Proof. Like that of Theorem 9.13.

\section{Quasirings.}

Definition 10.1. A quasiring is a collection of distinct quasigroups $f_{1}, \cdots, f_{k}, k \geq 2$, defined on the same set, such that for $i \neq j, 1 \leq i, j \leq k, f_{j}$ is left-distributive over $f_{i}$, i.e., $f_{j}\left(a, f_{i}(b, c)\right)=f_{j}\left(f_{i}(a, b), f_{i}(a, c)\right)$. Each $f_{j}$ is clearly idempotent.

TheOREM 10.2. There exist no quasirings of order $n \equiv 2(\bmod 4)$.

Proof. This is a consequence of Corollary 9.11.

THEOREM 10.3. There exist quasirings of orders $n \equiv 0,1,3(\bmod 4), n>3$ consisting of two quasigroups $f_{1}, f_{2}$, such that for all $i, j, 1 \leq i, j \leq 2, f_{i}$ is left-and right-distributive over $f_{j}$.

Proof. Let $f_{1}$ be a left- and right-distributive noncommutative quasigroup of order $n$; for example, one of the nonabelian quasigroups of order $n$ constructed in the proof of Theorem 9.8. Let $f_{2}=(12) f_{1}$. Clearly, $f_{1}$ is left- and right-distributive over itself, $i=1,2$. Typical of the proofs of the remaining cases is the computation showing that $f_{1}$ is left-distributive over $f_{2}$. 
For simplicity, denote $f_{1}(a, b)$ by $a b$ and $f_{2}(a, b)$ by $a \circ b$. The assertion $a(b \circ c)=(a b) \circ(a c)$ is equivalent to the assertion $b \circ c=p, a p=q, a b=r$, $a c=s$, imply $r \circ s=q$. This is equivalent to the assertion that $c b=p, a p=q$, $a b=r, a c=s$ imply $s r=q$. This in turn is equivalent to $c b=p, a b=r, a c=s \mathrm{im}$ ply $a p=s r$, which is equivalent to $a \cdot c b=a c \cdot a b$, left-distributivity of $f_{1}$.

COROLlary 10.4. The quasigroup $f$ is left-distributive if and only if (12) $f$ is left-distributive over $f$.

\section{Medial algebras}

\section{Constraints related to mediality.}

Definition 11.1. An algebra is medial if it satisfies the identity $a b \cdot c d$ $=a c \cdot b d$.

THEOREM 11.2. An algebra with two-sided unit is medial if and only if it is abelian and associative.

Proof. Let $e$ be the unit of a medial algebra. Then $e b \cdot c e=e c \cdot b e$; thus $b c=c b$, or the algebra is abelian. Also $a e \cdot c d=a c \cdot e d$; thus $a \cdot c d=a c \cdot d$, or the algebra is associative.

Conversely, let an algebra be associative and abelian. Then $a b \cdot c d$ $=(a b \cdot c) d=(c \cdot a b) d=(c a \cdot b) d=(a c \cdot b) d=a c \cdot b d$.

Corollary 11.3. An associative abelian algebra is medial.

Proof. This is proved in the proof of Theorem 11.2.

Theorem 11.4 (Hosszu [43]). An algebra satisfying the identity $a \cdot b c=c \cdot b a$ is medial.

Proof. $a b \cdot c d=d(c \cdot a b)=d(b \cdot a c)=a c \cdot b d$.

Theorem 11.5 (Hosszu [43]). If $Q$ is a quasigroup satisfying $a b \cdot c=b \cdot c a$ then $Q$ is a commutative group, hence medial.

Proof. Assume $e, x \in Q$ satisfy $e x=x$. Then $x(y e)=(e x) y=x y$. Cancellation yields $y e=y$ for all $y$. Thus $e$ is independent of $x$. Hence $e x=x$ for all $x$. Also $a(b \cdot c d)=a(d b \cdot c)=c a \cdot d b=(b \cdot c a) d$. If $d=e$ then $a \cdot b c=b \cdot c a$. But $b \cdot c a=a b \cdot c$. Thus $a \cdot b c=a b \cdot c$. Setting $c=e$ in $a b \cdot c=b \cdot c a$ yields $a c=c a$.

Theorem 11.6. An abelian quasigroup satisfying the identity $a b \cdot a c=d b \cdot d c$ is medial.

Proof. It shall first be shown that $a b=c d$ implies $a d=c b$. Let $a b=c d$ and $a d=c x$. Then $a b \cdot a d=c d \cdot c x$; thus $c b \cdot c d=c d \cdot c x$. Hence $c d \cdot c b=c d \cdot c x$, implying $x=b$.

Now assume $a b \cdot c d=a c \cdot b x$. Then $a b \cdot b x=a c \cdot c d$. Thus $b a \cdot b x=c a \cdot c d$, implying $c a \cdot c x=c a \cdot c d$ and hence $x=d$. 
Theorem 11.7. A group is medial if and only if it is abelian.

Proof. This is a consequence of Theorem 11.2.

Corollary 11.8. A quasigroup $Q$ satisfying the identities $a b \cdot a c=b c$ and $a b \cdot b=a$ is medial.

Proof. As will be shown in $\$ 12$ the constraint of mediality is invariant. If $\sigma=(23)$ then, as Tables 5.4 and 8.1 indicate, $\sigma Q$ is an abelian group. Theorem 11.2 and Corollary 6.13 thus prove the corollary.

Alternate proof. It may be illuminating to present the direct proof of Corollary 11.8 provided by the conjugation of the second part of the proof of Theorem 11.2.

The proof of Theorem 11.2 can be decomposed into atomic steps in the following way. For all $a, b, c, d$ define $x, y, z$ by $a b=x, c d=y, x y=z$. Define $t$ by $x c=t$. Then $t d=z$. But $c x=t$. Define $p$ by $c a=p$. Then $p b=t$. But $a c=p$. Define $q$ by $b d=q$. Then $p q=z$. This reduces to $a b \cdot c d=a c \cdot b d$.

Conjugation of this proof by $\sigma=(23)$ is the following. For all $a, b, c, d$ define $x, y, z$ by solving $a x=b, c y=d, x z=y$. Define $t$ by solving $x t=c$. Then $t z=d$. But $c t=x$. Define $p$ by solving $c p=a$. Then $p t=b$. But $a p=c$. Define $q$ by $b q=d$. Then $p z=q$.

From this proof mediality can be deduced by use of the computation observations in the Commentary on $\S 5$. To show $a p \cdot x z=a x \cdot p z$, define $c, y, d, t$, $b, q$ by use of the above equations. Observe that the last two sentences of the proof can be inverted to read: "define $q$ by $p z=q$. Then $b q=d$." Thus for all $a, p, x, z, a p \cdot x z=a x \cdot p z$.

Presumably, any proof of Corollary 11.8 would be more indirect than the proof of Theorem 11.2 since the conjugation by $\sigma=(23)$ of "evaluate" is "solve.".

The following lemma, theorem and proof is a recasting into the terminology of quasigroups of Knaster [36].

THeORem 11.9. An abelian left-distributive quasigroup possessing a linear ordering compatible with the algebra $(x<y$ implies ax $<a y)$ is medial.

The existence of the linear ordering is used only in the proof of the following lemma. As will be noted the lemma would also follow from incompatibility $(x<y$ implies $a x>a y)$.

LEMma 11.10. Under the above assumption if

$$
\begin{aligned}
& A=x y=B x \cdot C y, \\
& C=z t=A z \cdot B t
\end{aligned}
$$

then

$$
A=B(=C) \text {. }
$$


Proof of Lemma 11.10. The equations $x y=A x \cdot A y, z t=C z \cdot C t$ follow immediately. Thus

$$
A x \cdot A y=B x \cdot C y \text { and (2) } C z \cdot C t=A z \cdot B t \text {. }
$$

Assume $B<A$. Then by (1) $A<C$, so $B<A<C$. But by (2) $A<C$ implies $C<B$, so $A<C<B$. This is a contradiction, unless $A=B(=C)$.

Proof of Theorem 11.9. To show $a b \cdot c d=a c \cdot b d$, observe that

$$
a b \cdot c d=(a \cdot c d)(b \cdot c d)=(a c \cdot a d)(b c \cdot b d)=(a c \cdot d a)(c b \cdot b d)
$$

and

$$
\begin{aligned}
a b \cdot c d & =(a b \cdot c)(a b \cdot d)=(a c \cdot b c)(a d \cdot b d)=(a c \cdot c b)(d a \cdot b d) \\
& =(a c(d a \cdot b d)) \cdot(c b(d a \cdot b d))=((a c \cdot d a)(a c \cdot b d))((c b \cdot d a)(c b \cdot b d)) .
\end{aligned}
$$

Also

$$
c b \cdot d a=(c b \cdot d)(c b \cdot a)=(c b \cdot d)(a \cdot b c)=(c d \cdot b d)(a b \cdot a c)
$$

and

$$
\begin{aligned}
c b \cdot d a & =(c \cdot d a)(b \cdot d a)=(c d \cdot c a)(b d \cdot b a)=(c d \cdot a c)(b d \cdot a b) \\
& =((c d \cdot a c) \cdot b d) \cdot(a b(c d \cdot a c))=(c d \cdot b d)(a c \cdot b d)) \cdot(a b \cdot c d)(a b \cdot a c)) .
\end{aligned}
$$

Now let

$$
x=a c \cdot d b, \quad y=c b \cdot b d, \quad z=a b \cdot b c, \quad t=c d \cdot b d
$$

and

$$
A=a b \cdot c d, \quad B=a c \cdot b d, \quad C=c b \cdot d a .
$$

Then the equalities derived above can be written

$$
\begin{aligned}
& A=x y=x B \cdot C y, \\
& C=t z=t B \cdot A z .
\end{aligned}
$$

Commutativity and Lemma 11.10 yield $A=B$, that is, $a b \cdot c d=a c \cdot b d$.

12. Constraints equivalent to mediality. This section is devoted primarily to alternative descriptions of the constraint of mediality.

TheOREM 12.1. The identity $a b \cdot c d=a c \cdot b d$ is invariant. In fact, it may be characterized as the only identity of the form $a b \cdot c d=a^{\prime} b^{\prime} \cdot c^{\prime} d^{\prime}$, where $a^{\prime}, b^{\prime}, c^{\prime}, d^{\prime}$ is a permutation of $a, b, c, d$ which is invariant (other than the tautologous identity corresponding to the identity permutation).

Proof. If $\gamma$ is the constraint of mediality then so is (23) $\gamma$. For $\gamma$ is equivalent to

$$
a b=p, c d=q, p q=r, a c=s, b d=t \text { imply } s t=r \text { for all } a, b, c, d .
$$

Or equivalently 


$$
a b=p, c d=q, p q=r, a c=s, b d=t \text { imply } s t=r \text { for all } a, p, r, s .
$$

This in turn is equivalent to

$$
a b=p, p q=r, a c=s, s t=r, c d=q \text { imply } b d=t \text { for all } a, p, r, s .
$$

Conjugation of this constraint by $\sigma=(23)$ yields

$$
a p=b, p r=q, a s=c, s r=t, c q=d \text { imply } b t=d \text { for all } a, p, r, s .
$$

This reduces to the identity $a s \cdot p r=a p \cdot s r$, that is, (23) $\gamma \equiv \gamma$.

Obviously, (12) $\gamma \equiv \gamma$. Thus $\gamma$ is invariant.

A mechanical check of the identities of the form $a b \cdot c d=a^{\prime} b^{\prime} \cdot c^{\prime} d^{\prime}$ shows that only the two mentioned in the statement of Theorem 12.1 are invariant.

Theorem 12.2. A quasigroup $Q$ is medial if and only if it satisfies the constraint $\gamma: a b=a^{\prime} b^{\prime}, c d=c^{\prime} d^{\prime}$ imply $a c \cdot b d=a^{\prime} c^{\prime} \cdot b^{\prime} d^{\prime}$.

Proof. Let $Q$ be medial and satisfy the hypotheses of $\gamma$. Then $a b \cdot c d$ $=a^{\prime} b^{\prime} \cdot c^{\prime} d^{\prime}$ and hence $a c \cdot b d=a^{\prime} c^{\prime} \cdot b^{\prime} d^{\prime}$.

Conversely, let $Q$ satisfy $\gamma$. To show $a b \cdot c d=a c \cdot b d$ define $u$ by $b d=c u$. Then $a c=a c$ and $b d=c u$. Application of $\gamma$ yields $a b \cdot c d=a c \cdot c u=a c \cdot b d$.

Theorem 12.3. A quasigroup $Q$ is medial if and only if it satisfies the constraint $\gamma: p q=r s$ implies $x p \cdot y q=x r \cdot y s$.

Proof. Let $Q$ be medial and $p q=r s$. Then $x p \cdot y q=x y \cdot p q=x y \cdot r s,=x r \cdot y s$.

Conversely, let $Q$ satisfy $\gamma$ and $a, b, c, d \in Q$. Define $s$ by $b d=c s$. Then $a b \cdot c d=a c \cdot c s=a c \cdot b d$.

Theorem 12.4. A quasigroup $Q$ is medial if and only if it satisfies the two constraints

$$
x a=b y, z a=b w \text { imply } x w=z y
$$

and

$$
\text { The simultaneous equations } a b \cdot x=y \cdot b c \text {, }
$$

$$
a d \cdot x=y \cdot d c \text { have a solution } x, y .
$$

Proof. Assume $Q$ is medial and $x a=b y, z a=b w$. Then $x w \cdot a b=x a \cdot w b$ $=b y \cdot w b=b w \cdot y b=z a \cdot b y=z y \cdot a b$. Cancellation of $a b$ yields (12.5). Since $Q$ is medial (12.6) has the solution $x=b c, y=a b$.

Conversely, assume that $Q$ satisfies (12.5) and (12.6). To show $a b \cdot c d$ $=a c \cdot b d$ solve the simultaneous equations $a b \cdot x=y \cdot b d$ and $a c \cdot x=y \cdot c d$ for $x, y$. Application of $(12.5)$ yields $a b \cdot c d=a c \cdot b d$.

REMARK. Either an associative, left-distributive, right-distributive or medial algebra $A$ satisfies (12.6). The solutions $x, y$ are respectively, $(c, a)$, $(a c, a),(c, a c)$, and, as already noted, $(b c, a b)$. 
Definition 12.7. If $X$ is a set and $A$ is an algebra and $f, g: X \rightarrow A$, then the product $f g$ is defined by

$$
f g(x)=f(x) \cdot g(x) \text { for all } x \in X .
$$

Theorem 12.8. An algebra $A$ is medial if and only if for any algebra $B$ and homomorphisms $f, \mathrm{~g}: B \rightarrow A, f g$ is again a homomorphism.

Proof. Let $A$ be medial and $f, g: B \rightarrow A$ be homomorphisms. For $x, y \in B$, $f g(x y)=f(x y) g(x y)=(f(x) f(y))(g(x) g(y))=(f(x) g(x))(f(y) g(y))=f g(x) f g(y)$. Thus the product of two homomorphisms is again a homomorphism.

Assume that the product of any two homomorphisms into $A$ is again a homomorphism. Let $a, b, c, d$ be in $A$. Let $B$ be the free algebra on two generators $x, y$. Let $f: B \rightarrow A$ be the homomorphism defined by $f(x)=a, f(y)=b$ and $g: B \rightarrow A$ be similarly defined by $g(x)=c, g(y)=d$.

Since $f g$ is a homomorphism

$$
a b \cdot c d=f(x y) g(x y)=f g(x y)=f g(x) \cdot f g(y)=(f(x) g(x))(f(y) g(y))=a c \cdot b d .
$$

\section{Consequence of mediality.}

Definition 13.1. If $B$ is an algebra and $A$ is a medial algebra then Hom $(B, A)$ is the (medial) algebra of homomorphisms from $B$ into $A$, where the product is given by Definition 12.7 .

THEOREM 13.2. If $B$ is an algebra and $Q$ is a medial quasigroup then $\operatorname{Hom}(B, Q)$ is a (possibly void) medial quasigroup.

Proof. The only point of interest is the fact that if $f, g: B \rightarrow Q$ are homomorphisms and $h: B \rightarrow Q$ is defined by $f(x)=h(x) g(x)$ then $h$ is a homomorphism.

That $h(x y)=h(x) \cdot h(y)$ is a consequence of the equations:

and

$$
f(x y)=h(x y) \cdot g(x y)
$$

$$
\begin{aligned}
f(x y) & =f(x) f(y)=(h(x) g(x))(h(y) g(y))=(h(x) h(y))(g(x) \cdot g(y)) \\
& =(h(x) \cdot h(y))(g(x y)) .
\end{aligned}
$$

THEOREM 13.3. If $B$ is an algebra and $A$ is a medial algebra and $a \in A$ is an idempotent element, then the constant function $f: B \rightarrow A$ defined by $f(x)=a$ is a homomorphism.

Corollary 13.4. If $A$ contains an idempotent element $\operatorname{Hom}(B, A)$ is not empty.

Corollary 13.5. The algebra $A$ is idempotent if and only if for each algebra $B$, each constant function $f: B \rightarrow A$ is a homomorphism.

THEOREM 13.6. Any subquasigroup $N$ of a medial quasigroup $Q$ is normal in $Q$ in the sense that for each $a, b \in Q, a N \cdot b N=a b \cdot N$ and $N a \cdot N b=N \cdot a b$. 
Proof. If $m, n \in N$, then $a m \cdot b n=a b \cdot m n$. Thus $a N \cdot b N \subset a b \cdot N$.

Conversely, let $n \in N$. Represent $n=n_{1} n_{2}, n_{1}, n_{2} \in N$. Thus

$$
a b \cdot n=a b \cdot n_{1} n_{2}=a n_{1} \cdot b n_{2} \text { and } a b \cdot N \subset a N \cdot b N .
$$

The remainder of the theorem is proved similarly.

TheOREM 13.7. Let $N$ be a subquasigroup of the medial quasigroup $Q$ and $a, b \in Q$. Then, if $a N \cap b N \neq \phi, a N=b N$. Also if $N a \cap N b \neq \phi, N a=N b$.

Proof. Since $a N \cap b N \neq \phi$ there are $n_{1}, n_{2} \in N$ such that $a n_{1}=b n_{2}$. Define $c$ by $a=b c$. Then

$$
b c \cdot N=b c \cdot n_{2} N=b n_{2} \cdot c N=a n_{1} \cdot c N=a c \cdot n_{1} N=a c \cdot N,
$$

and thus

$$
b c \cdot N=a c \cdot N .
$$

Let $n_{0} \in N$ be a fixed element of $N$. Then

$$
a N \cdot c n_{0}=a c \cdot N n_{0}=a c \cdot N=b c \cdot N=b c \cdot N n_{0}=b N \cdot c n_{0} .
$$

Cancellation of $c n_{0}$ yields $a N=b N$.

Theorem 13.8. If $N$ is a subquasigroup of a medial quasigroup $A$ then the cosets $\{a N\}$ are a medial quasigroup under the law $a N \cdot b N=a b \cdot N . N$ is idempotent in this quasigroup.

Proof. This may be deduced from Theorems 13.6 and 13.7. Clearly $N$ is idempotent.

Theorem 13.9. An idempotent medial algebra is left-and right-distributive.

Proof. $a b \cdot a c=a a \cdot b c=a \cdot b c$. Similarly for right-distributivity.

THEOREM 13.10. The center of a medial quasigroup $Q$ is either empty or else all of $Q$.

Proof. Let $a$ be in the center of $Q$. Then $b b \cdot a x=b b \cdot x a$ for all $x, b \in Q$. Thus, $b a \cdot b x=b x \cdot b a$ for all $x, b$. Since for arbitrary $u, v \in Q$ the simultaneous equations $u=b a, v=b x$ may be solved for $b$ and $x, u v=v u$. Hence, $Q$ is abelian.

Theorem 13.11 (Frink [44, p. 704]). If $A$ is a medial algebra and $S$ and $T$ are subalgebras of $A$ then $U=\{s t \mid s \in S, t \in T\}$ is a subalgebra of $A$.

Proof. Let $s, s^{\prime} \in S$ and $t, t^{\prime} \in T$. Then $s t \cdot s^{\prime} t^{\prime}=s s^{\prime} \cdot t t^{\prime}$. So $U U \subset U$.

14. Examples of medial algebras. In addition to abelian groups there is a variety of sources of medial algebras. We present several examples, most of which have appeared elsewhere.

Example 1. In the terminology of Definition $9.10 A(G(n), p, q)$ is a medial 
algebra. If $(p, n)=1=(q, n)$ then $A$ is a quasigroup. If $p+q \equiv 1(\bmod n)$ then $A$ is idempotent. A similar construction can be made with $A\left(G F\left(2^{k}\right), a, \beta\right)$.

Example 2. (Murdoch [21], Toyoda [22], Bruck [26], Frink [44]). Let $G$ be an arbitrary group, $k \in G$ be fixed, and $S, T: G \rightarrow G$ be commuting automorphisms. Then the quasigroup defined by $a \circ b=k S(a) T(b)$ is medial. Example 1 is a special case of this.

EXAMPLE 3 (Aczel [31]). Let $R$ be a commutative ring and $f: R \rightarrow R$ be an equivalence and $p, q, t \in R$, fixed. Define

$$
a \circ b=f^{-1}(p f(a)+q f(b)+t) .
$$

If $R$ is a field and $p q \neq 0$ then this medial algebra is also a quasigroup. The following theorem is pertinent here.

Theorem 14.2 (Aczel [31]). A continuous medial quasigroup on the space $X$ of real numbers must be of the form 14.1, where $f$ is a homeomorphism of $X$.

Example 4 (Sholander [35]). Let $E_{2}$ be the Euclidean plane. Define an algebra on $E_{2}$ by setting $x \cdot y=$ midpoint of the segment $x y$. That this quasigroup is medial is equivalent to the theorem asserting that the midpoints of the sides of a quadrilateral are the vertices of a parallelogram. (This example is simply the direct product of two copies of Example 3 with $f=$ identity, $p=1 / 2=q, t=0$.)

EXAmple 5 (Sholander [35]). Let $A B C$ be a fixed oriented triangle in $E_{2}$. If $x, y \in E_{2}$ define $x y=z$ by the demand that the triangle $x y z$ is similar to $A B C$ and has the same orientation (

EXAmPLE 6 (Sholander [35]). Let $s$ be a fixed number of the real projective line $P_{1}$ and $r$ a real number. For $x, y \in P_{1}$ define $x y=z$ by the demand $R(s, x, y, z)=r$. This example generalizes $(x+y) / 2$ (a special case of Example 3$)$, since the midpoint is the harmonic conjugate of the point at infinity.

EXAMPLE 7 (Mituhisa [25]). On $C$, the circumference of a circle, define $x y=z$ if $z$ is the mirror image of $y$ in the diameter through $x$.

EXAMPLE 8 (Mituhisa [25]). On $P$, a parabola, define $x y=z$ by the demand that $z \in P$ and that the line $y z$ is parallel to the tangent at $x$ (set $x x=x$ ). This turns $P$ into a medial quasigroup.

EXAMPLE 9. Let $C$ be a conic in the real projective plane and $L$ a line not meeting $C$. If $x, y \in C$ define $x y=z$ by the demand that $z \in C, z \neq y$, and that $L$, the tangent to $C$ at $x$, and the line $z y$ be concurrent (set $x x=x$ ). That this defines a medial algebra on $C$ may be proved in the following way. If $C$ is a circle and $L$ the line at infinity this is simply Example 6. An appropriate projectivity shows that the more general construction produces a medial algebra.

EXAMPLE 10. Let $C$ be a conic in the real projective plane and $L$ a tangent to $C$. Let $Q$ denote $C$ minus the point of contact of $L$. If $x, y \in Q$ define $x y=z$ by the demand that $z \in Q, z \neq y$ and that $L$, the tangent at $x$, and the line $z y$ 
be concurrent (set $x x=x$ ). Since this is the limiting case of the preceding example continuity yields that $Q$ is a medial quasigroup. (This generalizes Example 8).

Applying $\sigma=(13)$ to the $Q$ of Example 10, one deduces the following theorem.

THEOREM 14.3. Let $Q$ denote a conic minus the point of contact of a tangent $L$. If $x, y \in Q$ define $x y=z$ by the demand that $L$, the tangent at $z$, and the line $x y$ be concurrent (set $x x=x$ ). Then $Q$ is a medial, abelian, idempotent quasigroup.

This suggests the following theorem.

THEOREM 14.4. Let $K$ denote a smooth convex closed curve without line segments minus the point of contact of a tangent L. If $x, y \in K$ define $x y=z$ by the demand that $L$, the tangent at $z$, and the line $x y$ be concurrent (set $x x=x$ ). Then $K$ is an abelian idempotent quasigroup. It is medial if and only if the curve is a conic.

EXAmple 11. Let $C$ be a line conic in the real projective plane and $F$ a point of contact of $C$. Let $Q$ denote $C$ minus the tangent at $F$. If $x, y \in Q$ define $x y=z$ be the demand that $F$, the point of contact of $z$, and intersection of $x$ and $y$ be collinear ( $\operatorname{set} x x=x$ ). Then $Q$ is a medial, abelian, idempotent quasigroup (it is simply the projective dual of the quasigroup of Theorem 14.3).

(Moreover, it can be shown that Example 11 is isomorphic to the quasigroup of Theorem 14.3; in fact, the function which assigns to each tangent its point of contact is an isomorphism.)

\section{ORTHOGONAL ALGEBRAS}

\section{Implication of orthogonality on an algebra.}

Definition 15.1. Two algebras $f, g$ defined on the same set $X$ are orthogonal if

$$
f \times g: X \times X \rightarrow X \times X
$$

defined by $f \times g\left(x, x^{\prime}\right)=\left(f\left(x, x^{\prime}\right), g\left(x, x^{\prime}\right)\right)$ is an equivalence. The algebra $g$ is an orthogonal complement to $f$.

For $X$ finite $f$ and $g$ are orthogonal if and only if $f \times g$ is onto (or $f \times g$ is one-one). For arbitrary $X, f$ and $g$ are orthogonal if and only if: for every $a, b \in X$ the simultaneous equations $f(x, y)=a, g(x, y)=b$ have a unique solution $x, y$.

THEOREM 15.1. A finite commutative algebra $A$ is a quasigroup if and only if it is orthogonal to the algebra $A^{*}$ whose multiplication is given by $x * y=x \cdot x y$.

Proof. Let $A$ be a quasigroup and $a, b \in X$. Then there exists $x$ so $x a=b$, and then $y$ so $x y=a$. Thus, the simultaneous equations $x y=a, x * y=b$ have a solution $x, y$. 
Conversely, let $A$ be orthogonal to $A^{*}$. Assume $x y=x z$. Then $x \cdot x y=x \cdot x z$, or $x * y=x * z$, thus $y=z$. Since $A$ is commutative it is a quasigroup.

THEOREM 15.2. Every quasigroup has an orthogonal complement (which is not necessarily a quasigroup).

Proof. Similar to the first part of the proof of Theorem 15.1.

16. Constraints implying orthogonality. This section presents various methods of associating with a quasigroup a few specific algebras as candidates for orthogonal quasigroup complements.

Theorem 16.1. A quasigroup $Q$, satisfying the constraint $x \cdot x z=y \cdot y z$ implies $x=y$, possesses an orthogonal complement which is a quasigroup.

Proof. It is sufficient to show that $Q^{*}$, defined in the statement of Theorem 15.1 , is a quasigroup. The equation $s * z=x * y$ is equivalent to $x \cdot x z=x \cdot x y$, which, since $Q$ is a quasigroup, implies $y=z$. The equation $z * x=y * x$ is equivalent to $z \cdot z x=y \cdot y x$, which by assumption implies $y=z$.

THEOREM 16.2. There exist quasigroups satisfying the constraint $x \cdot x z=y \cdot y z$ implies $x=y$ for orders $n \equiv 0,1,3(\bmod 4)$.

Proof. If $\alpha \beta(1+\beta) \neq 0$ and $k \geqq 2$ then $A\left(G F\left(2^{k}\right), \alpha, \beta\right)$ satisfies the constraint. Direct product of these with abelian groups of odd order proves the theorem.

TheOREM 16.3. A quasigroup satisfying the identity $a \cdot a b=b a$ has an orthogonal complement.

Proof. This is a consequence of Theorem 16.1.

THEOREM 16.4. Let $Q$ be a finite quasigroup and $\sigma \in S_{3}$. Then $Q$ is orthogonal to $\sigma Q$ if and only if $Q$ satisfies the constraint $\gamma$, depending on $\sigma$ as is indicated by the following table.

\begin{tabular}{l|ll}
\multicolumn{1}{c|}{$\sigma$} & $\gamma$ \\
\hline$(12)$ & $a b=a^{\prime} b^{\prime}, b a=b^{\prime} a^{\prime}$ & imply $a=a^{\prime}, b=b^{\prime}$ \\
$(13)$ & $a b \cdot b=a c \cdot c$ & implies $b=c$ \\
$(23)$ & $b \cdot b a=c \cdot c a$ & implies $b=c$ \\
$(13)$ & $b \cdot a b=c \cdot a c$ & implies $b=c$ \\
$(132)$ & $b a \cdot b=c a \cdot c$ & implies $b=c$ \\
\hline
\end{tabular}

Proof. The case $\sigma=(13)$ is typical. Designate by $*$ the algebra of $\sigma Q . Q$ is orthogonal to $\sigma Q$ if and only if the simultaneous equation $x y=a, x * y=b$ have a solution $x, y$ for all $a, b$. In $Q$ this translates into the demand that the equations $x y=z, b y=x$ have solutions or equivalently $b y \cdot y=a$ has a solution. Since $Q$ is finite, this is equivalent to the constraint $\gamma$ listed in the table.

Theorem 16.6. Let $Q$ be a finite quasigroup and $\sigma, \tau \in S_{3}, \sigma \neq \tau$. Then $\sigma Q$ is 
orthogonal to $\tau Q$ if and only if $Q$ satisfies the constraint listed in the following table.

(16.7)

\begin{tabular}{|c|c|c|c|c|}
\hline & (13) & (23) & (123) & (132) \\
\hline (12) & $b \cdot a b=c \cdot a c \Rightarrow b=c$ & $b a \cdot b=c a \cdot c \Rightarrow b=c$ & $a b \cdot b=a c \cdot c \Rightarrow b=c$ & $b \cdot b a=c \cdot c a \Rightarrow b=c$ \\
\hline (13) & & $\begin{array}{l}\quad(1) \\
a \cdot x b=x \\
\text { has solution }\end{array}$ & $\begin{array}{l}a \cdot b x=x \\
\text { has solution }\end{array}$ & $\begin{array}{l}a x=x b \\
\text { has solution }\end{array}$ \\
\hline (23) & & & $\begin{array}{l}x a=b x \\
\text { has solution }\end{array}$ & $\begin{array}{l}x b \cdot a=x \\
\text { has solution }\end{array}$ \\
\hline (123) & & & & $\begin{array}{l}\quad(1) \\
a \cdot x b=x \\
\text { has solution }\end{array}$ \\
\hline
\end{tabular}

(1) Equivalently $a x \cdot b=x$ has solution.

Proof. Like that of preceding theorem.

COROLLARY 16.8. If a finite quasigroup of order $n$ satisfies at least one of the constraints in (16.5) and (16.7) then there is a pair of orthogonal quasigroups of order $n$.

COROLlaRy 16.9. If a finite quasigroup of order $n$ satisfies all of the constraints in (16.5) and (16.7) then there are six mutually orthogonal quasi-groups of order $n$.

\section{Conjugates of various constraints}

17. Examples of invariant constraints. The following identities are invariant: $a b \cdot c d=a c \cdot b d ; a b \cdot c a=a c \cdot b a ; a a=a ; a b \cdot b a=a ; a b \cdot b c=a c$.

The following constraints are invariant: $a b \cdot c a=a$ implies $a c \cdot b a=a$; $c a=b d, e a=b f$ imply $c f=e d ; a b=a^{\prime} b^{\prime}, a c=a^{\prime} c^{\prime}, b d=b^{\prime} d^{\prime}$ imply $c d=c^{\prime} d^{\prime}$.

18. Examples of noninvariant constraints. The list of conjugates of associativity and commutativity is to be found in (8.1) and (9.2). The following table is a list of conjugates of some other constraints that have been considered in the literature.

\begin{tabular}{|c|c|c|c|c|c|}
\hline$I$ & (12) & (13) & (23) & (123) & (132) \\
\hline$a b \cdot b a=b$ & $a b \cdot b a=b$ & $\begin{array}{l}a \cdot b c=c \Rightarrow \\
b c \cdot b=a\end{array}$ & $\begin{array}{l}a b \cdot c=a \Rightarrow \\
b \cdot a b=c\end{array}$ & $\begin{array}{l}a \cdot b c=c \Rightarrow \\
b c \cdot b=a\end{array}$ & $\begin{array}{l}a b \cdot c=a \Rightarrow \\
b \cdot a b=c\end{array}$ \\
\hline$a \cdot b c=c a \cdot b$ & $a \cdot b c=c a \cdot b$ & $(a \cdot b c) a b=c$ & $((a b \cdot c) a) b=c$ & $(a \cdot b c) c b=c$ & $((a b \cdot c) a) b=c$ \\
\hline $\begin{array}{l}a b \cdot c=a d \Rightarrow \\
e b \cdot c=e d\end{array} \Rightarrow$ & $\begin{array}{l}c \cdot b a=d a \Rightarrow \\
c \cdot b e=d e\end{array}$ & $\begin{array}{l}a b \cdot c=a d \Rightarrow \\
e b \cdot c=e d\end{array}$ & $\begin{aligned} c a & =c^{\prime} b \\
d a & =d^{\prime} b \\
c d & =c^{\prime} d^{\prime}\end{aligned}$ & $\begin{array}{l}c a=c^{\prime} b \\
d a=d^{\prime} b \\
c d=c^{\prime} d^{\prime}\end{array}$ & $\begin{array}{l}a \cdot b c=d c \Rightarrow \\
a \cdot b e=d e\end{array}$ \\
\hline$a b \cdot a c=d b \cdot d c$ & $c a \cdot b a=c d \cdot b d$ & $\begin{array}{l}a x \cdot b=x c \Rightarrow \\
a y \cdot b=y c\end{array}$ & $\begin{array}{l}a b=c d \Rightarrow \\
a \cdot b x=c \cdot d x\end{array}$ & $\begin{array}{l}a b=c d \Rightarrow \\
x a \cdot b=x c \cdot d\end{array}$ & $\begin{array}{l}a \cdot x b=c x \Rightarrow \\
a \cdot y b=c y\end{array}$ \\
\hline
\end{tabular}




\section{VARIETIES}

19. Application of conjugation to varieties. Let $A, Q, G, C$ denote the class of all algebras, quasigroups, groups and commutative groups respectively. Then $A \supset Q \supset G \supset C$.

Definition 19.1. A class $S$ of algebras is a variety in a class $T$ of algebras if there is an identity $\gamma$ such that $S$ consists of precisely those members of $T$ satisfying $\gamma$.

TheOREM 19.2. For $\sigma=I$ or (12), $\sigma G$ is not a variety in $A$ but for all other $\sigma, \sigma G$ is a variety in $A$.

The proof is to be found in Higman and Neumann [40].

THEOREM 19.3. If $\sigma \neq I$, (12) then the subclass of $\sigma G$ satisfying an identity $\gamma$ is a variety in $A$.

The proof is to be found in Higman and Neumann [40].

THEOREM 19.4. The class $G_{2}$ of groups in which every element is of order 2 is a variety in $A$.

Proof. Let $\sigma=(13)$. By Theorem $19.3 \sigma G_{2}$ is a variety in $A$. But, by Corollary $7.3, \sigma G_{2}=G_{2}$.

Theorem 19.5. If $\sigma \neq I$, (12) then $\sigma C$ is a variety in $A$.

Proof. Higman and Neumann [40] offer two proofs of this. The first consists of noting that it is a consequence of Theorem 19.3 and the second consists of presenting a short identity for $\sigma C$. For $\sigma=(13)$ this identity is $a(b c \cdot b a)=c$, which is $\sigma \gamma$, where $\gamma$ is the identity $a b \cdot c=b \cdot c a$ distinguishing $C$ as a variety in $Q$ (Theorem 11.5).

\section{Commentary}

20. Remarks. On §3. The phrase "Polyadic Group" was used by E. L. Post [18] who observed the similarity between the notions "single valued" and "unique solution." The observation that the symmetric group operates on graph to yield a new graph was exploited by Lefschetz $[23$, p. 175] in a neat presentation of the relation between cup and cap product.

On $\S 4$. That quasigroups come in sextuplets was observed most recently by $D$. A. Norton and the author in developing the theory of cycles $[46 ; 47]$ which is based directly on the graph $G$. Bruck $[26$, p. 24] pointed out the presence of the five additional quasigroups. H. W. Norton [16], in his table of quasigroups of order 7 lists those which are "identical with their adjugates" ("totally symmetric" in the sense of Definition 8.2). See in particular [16, pp. 272, 282-285]. Bruck investigated totally symmetric quasigroups, especially their relation to loops, in [26]. Several times, in the theory of groups, 
the quasigroups $(132) Q,(23) Q$ have been studied, especially in axiomatizing groups in terms of the inverse operations (e.g. $[5 ; 9 ; 11 ; 12 ; 14 ; 45]$ ).

As is well-known, Definition 4.2 of a group can be weakened to: $\pi_{1}, \pi_{2}$ are onto, $\pi_{3}$ is an equivalence and associativity holds. Various conditions which give rise directly or indirectly to groups have been studied. (1) As Theorem 5.4 indicates, there is a one-one correspondence between groups and quasigroups satisfying the identity $a b \cdot a c=b c$. (2) (Suschkewitsch [3]). If a quasigroup satisfies the constraint that the solution, $c$, of $x a \cdot b=x c$ is independent of $x$ (that is, $x a \cdot b=x c$ implies $y a \cdot b=y c$ (see third row of 18.1 and related $B$ of Lemma 7.5) then the quasigroup defined by $a \circ b=c$ is a group. (3) (Evans [37]). A loop for which there exist equivalences $P_{i}, Q_{i}, 1 \leqq i \leqq 5$, satisfying

$$
P_{5}\left(P_{1}(x) P_{4}\left(P_{2} y \cdot P_{3} z\right)\right)=Q_{5}\left(Q_{3}\left(Q_{1} x \cdot Q_{2} y\right) Q_{4} z\right)
$$

is a group. (4) Murdoch [21] obtains an abelian group from a medial quasigroup. If $Q$ is a medial abelian quasigroup and $a=a a$ is a fixed element of $Q$ then a special example of his method is the quasigroup given by $a x \circ a y=x y$. That this quasigroup is an abelian group is a consequence of the easily verified facts that it is a medial loop (with unit " $a$ ").

On $\S 5$. In the computation and simplification of conjugate constraints, the following observations are of aid. Usually the atomic form of a constraint takes the form $E_{1}, \cdots, E_{k}$ imply $E_{k+1}$ where

1. $E_{j}$ is an equation of the form $a b=c$ where $a, b, c$ are distinct variable $(1 \leqq j \leqq k+1) .2$. $E_{i}$ and $E_{j}$ have at most one variable in common $(i \neq j)$. 3 . Each variable appearing in the $E_{i}$ appears at least twice. 4 . For any $E_{i}, E_{j}$ there is a sequence $E_{i}=E_{k_{1}}, E_{k_{2}}, \cdots, E_{k_{n}}=E_{j}$ where $E_{k_{\alpha}}$ and $E_{k_{\alpha+1}}(1 \leqq \alpha$ $\leqq n-1)$ have a variable in common.

For such systems one can easily see that

(a) Any $k$ of the equations imply the remaining one,

(b) The universal operatory " $\wedge$ " can be assumed to run over all the variables or any subset of them. (If the variables in the subset, $S$ are "independent" and define the remaining variables by successive application of the $E_{j}$, then their number is independent of $S$ ).

The identity $a b \cdot a c=b c$ is to be found in Suschkewitsch [3, p. 213], Tarski $[12$, p. 254], Higman and Neumann [40] and Furstenberg [45].

Corollary 5.5 raises several questions. It shows that, though we define a function rigorously as a subset of $X^{k}$, we tend to look at $X^{k}$ along one preferred axis. In our bias, we develop a multiplicative notation based on operations with parentheses. Since we would like to dispense with parentheses, we demand associativity. As Corollary 5.6 shows, the study of associative quasigroups is equivalent to the study of quasigroups satisfying a rather uninviting identity.

The interpretation of associativity in the Cayley multiplication table (Andreas Speiser, Die Theorie von Gruppen von endlicher Ordnung, New York, 
Dover, 1943, p. 14) does not compare in simplicity to that of commutativity. On the other hand, associativity can be expressed in terms of commutativity. Specifically, an algebra is associative if its left and right translations commute with each other.

It would be illuminating to know what are the implications of associativity which give it such a prominence before all other identities. For example, if an algebra is a homomorphic image of a group it is a group. On the other hand, arbitrary quasigroups or even loops do not have this property [32]. The free group on $m$ generators is easily obtained from the free algebra on $m$ generators by "removing parentheses," adjoining a unit $e$, and introducing solutions to the $m$ equations $x g_{i}=e$, where $g_{i}$ is a generator. The free quasigroup on $m$ generators is obtained from the free algebra by introducing solutions to an infinity of equations [29]. There is an analogy of this situation to that of the algebraic closure of the real field and the rational field. Another implication of associativity is that the equivalence, $\phi$, defined by $a x=\phi(x) a$, is an automorphism. (This is also a consequence of other identities [see Theorem 9.12]).

On $\S 6$. The use of Lemma 6.3 is discussed in the paragraph following Corollary 6.14.

On $\$ 7$. Theorem 7.4 is due to Sade [38, p. 13]. Statements B, B' of Lemma 7.5 are some of Suschkewitsch's generalizations of associativity [3]. Another of his generalizations is: the solution " $d$ " to $a \cdot b c=a b \cdot d$ is independent of $b$. The case $d=a c$ is left-distributivity.

On $\S 8$. The identities $a \cdot a b=b$ and $b \cdot b a=a$ are called the left and right law of keys respectively, Mituhisa [25], Sade [38, p. 3]. The phrase "totally symmetric" is due to Bruck [26, p. 34]. The author has been informed that Theorem 8.5 also appears in an inaccessible paper by R. H. Bruck: $A$ note on Steiner triple systems, SCAMP working paper 1953. The proof is the same as in [47].

Conjugation of the well-known theorem that there are no commutative idempotent quasigroups of even order is the theorem: there are no quasigroups of even order satisfying the left (right) law of keys.

On §9. C. Burstin and W. Mayer studied quasigroups which are left-and right-distributive [4]. They stated that there are none of orders 2 and 6 , observed that the group of automorphisms is transitive, and showed that such a quasigroup is idempotent. A problem of Bourbaki [39, p. 62] is based on their paper. The only other places where self-distributivity was investigated seem to be in Mituhisa [25], Frink [44], Bruck [28] and in the study of functional equations in real variables (see $[31 ; 36 ; 41]$ ).

Since the relation $R$ is preserved under homomorphisms, it may be used as a local test for the nonexistence of homomorphisms from one quasigroup onto another. For example, if $x^{\prime} \in Q^{\prime}$ has an equivalence class with more members than any equivalence class defined on any $x \in Q$, then $Q^{\prime}$ cannot be the homomorphic image of $Q$. 
The construction of quasigroups from Galois fields dates back at least to Bose [13] 1933, and the construction based on abelian groups at least to Burstin and Mayer [4] 1929.

In groups the equivalences of Theorems 9.12 and 9.14 are automorphisms if and only if $a=b$.

On $\S 10$. A quasiring is remotely related to a lattice. It is also related to a structure introduced by Suschkewitch [7]. He considered two commutative, associative algebras with units such that each algebra distributes over the other. In his proofs he does not use associativity.

On $\$ 11$. The identity $a b \cdot c d=a c \cdot b d$ has been studied by Murdoch [21], Toyoda [22], Etherington [24], Bruck [26], Aczel [31], Knaster [36] Frink [44], under a variety of names. The word "medial" is appropriate for two reasons: (1) The middle two terms are interchanged in $a b \cdot c d$ to obtain $a c \cdot b d$ and (2) The arithmetic mean is a medial operation and most of the examples of $\$ 14$ are generalizations of this algebra. The connotation of "midness" is therefore not inappropriate. Since there are abelian nonmedial algebras, the name "quasi-abelian" which has been used is misleading.

Theorem 11.2 and Corollary 11.3 are to be found in Etherington [24], Bruck [26], Dubreil [42, p. 84]. Theorem 11.5 is proved in Hosszu [43]. Theorem 11.6 is stated in Sade $[38$, p. 13].

The author came across the work of Knaster, Aczel, and Hosszu quite by chance. It may well be that other work on functional equations in real variables may impinge on the theory of quasigroups.

Lemma 11.10 is not necessarily true for arbitrary left- and right-distributive quasigroups as the example $A(G(7), 3,5)$ shows.

On $\$ 12$. Constraint 12.5 and part of the proof of Theorem 12.4 is to be found in Toyoda [22]. Part of Theorem 12.8 is to be found in Frink [44, p. 701].

On $\S 13$. Theorem 13.3 is analogous to the theorem in topology asserting that any constant function is continuous. Two theorems of Bates and Kiokemeister [32] also have topological analogs. The analog of Lemma 3 of [32, p. 1184] is the theorem asserting that the number of sheets in covering space is well defined. The analogs of Lemma $5[32, \mathrm{p} .1184]$ are the various theorems on continuous functions with the property that $f^{-1}(y)$ is compact.

If one preferred to study only structures which were preserved under conjugation one would study quasigroups but not groups and idempotent quasigroups but not loops.

Weaker definitions of normality exist. Garrison [19] defines a subset $N$ of a finite quasigroup $Q$ to be normal in $Q$ if for every $a, b \in Q$ there is $c$ so $a N \cdot b N=c N$. Kiokemeister [30] defines a subset $N$ of a quasigroup $Q$ to be normal in $Q$ if there is an equivalence relation $R$ on $Q$ satisfying (1) $a R b$, $c R d \Rightarrow a c R b d$ (2) $a c R b c \Rightarrow a R b$ and (3) $c a R b a \Rightarrow c R b$ with $N$ as one of the equivalence classes of $R$. Both observe that their definitions are equivalent to the 
definition: there exists a quasigroup $Q^{\prime}$ and homomorphism $f: Q \rightarrow Q^{\prime}$, onto $Q^{\prime}$, with $N$ as preimage of an element of $Q^{\prime}$. This last definition readily shows that normality is invariant under conjugation, i.e., for $\sigma \in S_{3}, N$ is normal in $\sigma Q$ if $N$ is normal in $Q$. In fact $f$ is also a homomorphism from $\sigma Q$ onto $\sigma Q^{\prime}$. By letting $\sigma=(12)$ one obtains Garrison's theorem that the product of two right cosets is also a right coset. It is interesting to note that Kiokemeister's conditions (1), (2), (3), are not individually invariant under conjugation. If one wishes $N$ to be a normal subquasigroup then one could demand that $N$ be the preimage under $f$ of an idempotent element of $Q^{\prime}$.

Results and methods similar to Theorems 13.6, 13.7, 13.8 appear in Murdoch $[15 ; 20 ; 21]$, and Bruck [26].

On $\$ 14$. Curtis Fulton has obtained a purely projective synthetic proof of Theorem 14.2, and has pointed out that Example 6 yields an analytic proof since the algebra of $P_{1}$ may be introduced on $C$ itself.

Application of Murdoch's method (see Commentary on \$4) for constructing an abelian group from a medial quasigroup has some interesting geometric consequences. For example, if $C$ is a parabola, $L$ the tangent at infinity, and $a$ the vertex of $C$, then projection of the abelian group thus defined on $C$ from infinity upon the tangent at $a$, produces an abelian group $G$ on the tangent with unit " $a$ ". It is easy to see that $G$ is ordinary addition.

Addition on the line minus a point is usually defined projectively by the choice of one point on the line and three lines, a total of four arbitrary constructions. The medial quasigroup on a conic minus a point involves no arbitrary choice. In view of these facts, it is reasonable to suggest that the medial algebra on the conic in some respects is more "natural" than addition on the line.

On $\S 16$. Euler conjectured that there are no orthogonal quasigroups of order $4 k+2$. MacNeish [1, p. 221] conjectured that there are at most $a-1$ mutually orthogonal quasigroups of order $n$, where $a>1$ is the smallest divisor of $n$ such that $(a, n / a)=1$. He showed that the number $a-1$ is always assumed.

The constraint of Theorem 16.1 is the opposite of the identity $a \cdot a b=b$ in the sense that the identity $a \cdot a b=b$ is equivalent to the constraint: $a \cdot a b$ is independent of $a$. Indeed, assume $a \cdot a b$ is independent of $a$. Choose $c$ so $c b=b$. Then $c \cdot c b=c b=b$; so $a \cdot a b=b$ for all $a$.

Quasigroups satisfying $a \cdot a b=b a$ of Theorem 16.3 have several interesting properties. They are idempotent and distinct elements do not commute. Some models for this identity are

$$
\left(\begin{array}{llll}
a & c & d & b \\
d & b & a & c \\
b & d & c & a \\
c & a & b & d
\end{array}\right)
$$


and $A(G(n), p, a)$ where $p=q^{2},(2 q+1)^{2} \equiv 5(\bmod n)$ and $n$ is odd (such systems exist if and only if 5 is a quadratic residue of $n)$; also $A\left(G F\left(p^{k}\right), \alpha, \beta\right)$ can be used if $5^{\left(p^{k}-1\right) / 2}=1$. For example $(1,4,5,11,13,16) \subset J(a \cdot a b=b a)$ and $2,3,6 \notin J(a \cdot a b=b a)$.

One way to assure that the equation $a x=x b$ of Table 16.7 has a solution is to demand that the identity $a \cdot a b=a b \cdot b$ be satisfied. This identity is satisfied by $A\left(G F\left(2^{k}\right), \alpha, \beta\right)$ where $k \geq 2$ and $\alpha=1+\beta$ (hence $\beta=1+\alpha$ ), and $\beta \cdot(1+\beta) \neq 0$. Or, one could demand that the identity $a \cdot b a=b a \cdot b$ be satisfied. $A(G(n), p, q)$ can be constructed satisfying this identity if -1 is a quadratic residue of $n$ (i.e. $n$ is a product of primes of the form $4 k+1$ ) and $A\left(G F\left(p^{k}\right), \alpha, \beta\right)$ can be, if $p \neq 2$ and $(-1)^{\left(p^{k}-1\right) / 2}=1$.

On $\$ 17$. Observe the similarity of the last constraint of $\$ 17$ to Malcev's condition $a b=a^{\prime} b^{\prime}, a c=a^{\prime} c^{\prime}, d b=d^{\prime} b^{\prime}$, imply $d c=d^{\prime} c^{\prime}$ (Dubreil [42, p. 267]), which is a necessary condition that a semigroup be imbeddable in a group.

On $\S 18$. The identity $(a \cdot b c) a b=c$ has an unusual property. It is equivalent to the identity $a(b c \cdot b a)=c$, which cannot be deduced from it by cancellation or left or right multiplication. This is a counterexample to the conjecture: if a constraint reduces to an identity then is this identity unique (up to the obvious changes of variables, etc.)? That the two identities are equivalent can be proved in the following manner. The first is

$$
b c=x, a x=y, a b=z \text { imply } y z=c
$$

or equivalently

$$
y z=c, a x=y, a b=z \text { imply } b c=x
$$

which reduces to $x=b(a x \cdot a b)$, which is equivalent to the identity $a(b c \cdot b a)=c$.

The first condition of the third row of 18.1, due to Suschkewitsch [3], asserts that the product of two right translations is a right translation, hence generalizes associativity.

21. Questions and problems. The proposals to follow may probably run from the trivial to the impossible. They are intended primarily to indicate some avenues of future investigation.

1. If associativity and commutativity imply the invariant identity $\gamma$, does mediality imply $\gamma$ ?

2. Is there an identity belonging to the alternating group?

3. Is a left-distributive quasigroup right-distributive? ${ }^{(3)}$

4. Does any combination of the hypotheses: left-distributivity, rightdistributivity, commutativity imply mediality?

5. Can a quasigroup without idempotent elements and of order $4 k+2$ be homogeneous?

(3) J. Erdös and M. Hosszu have constructed an infinite cancellation algebra which is left, but not right, distributive. 
6. Discuss models for $a \cdot a b=b a, a \cdot a b=a b \cdot b, a \cdot b a=b a \cdot b$, in particular, their orders.

7. Show that none of the constraints of $\$ 16$ can be satisfied by a quasigroup of order $4 k+2$. (Or else use one to obtain a counter-example to Euler's conjecture.)

8. What medial algebra on the conic is related to the multiplicative group on the projective line (see Commentary on $\$ 14)\left({ }^{4}\right)$ ?

9. Let $y=f(x)$ be a differentiable function whose graph is a convex curve $K$ containing no line segments. If $P, Q \in K$ define $P \circ Q$ to be the point of $K$ whose tangent is parallel to $\overline{P Q}$ (set $P \circ P=P$ ). This quasigroup on $K$ is idempotent and abelian and satisfies all identities deducible from these properties. What other identities can this quasigroup satisfy? (E.g. $K$ is a conic if and only if it is medial.)

10. Is the class of groups satisfying the identity $x^{3}=1$ a variety in $A$ ?

\section{Added in Proof. Historical Addendum.}

References I to VII below, especially the work of Schröder, show that the theory of identities on quasigroups has a history going back to the last century.

Briefly:

I: vectors introduced as elements of quasigroup conjugate to abelian group of points in Euclidean space; identities $A B+B C=A C, A A=B B$ appear.

II : identities between (13) $(G)$ and $G$ studied.

III : notion of conjugate quasigroups appears (p. 305) and totally symmetric $Q$ (p. 306); nonidempotent models exhibited of all orders.

IV: finite and infinite idempotent models of the identity $c b(b \cdot a c)=a$ treated; model of order 8 shows conjecture in [47] is wrong; conjugate identities (p. 194); invariance of preceding identity under alternating group (thus answering question 2).

$\mathrm{V}$ : list of external symmetries of quasigr oups of orders 3 and 4.

VI: equivalence of totally symmetric $Q$ with $Q$-models of the identity $a \cdot a b=b c \cdot c$; identity $b a \cdot a c=b c$ implies associativity, mediality, total symmetry (p. 250); models of preceding identity of orders $2^{n}$ exhibited; mentions right distributivity.

VII $: a \cdot a b=b, a c \cdot b c=a c, a a=b b$ shown to be conjugate to constraints of abelian group (pp. 56, 59).

I. Hermann Grassman, Die lineale Ausdehnungslehre, Leipzig, 1844; 2 Aufl., 1878.

II. H. Hankel, Theorie der complexen Zahlensysteme, Leipzig, 1867.

III. E. Schröder, Rechnung mit Wurfen, Math. Ann. vol. 10 (1876) pp. 289-317.

(4) This question has been answered by Curtis Fulton and the author. 
IV. - Ueber eine eigentumliche Bextimmung einer Funktion durch formale Anforderungen, J. Reine Angew. Math. vol. 90 (1880) pp. 189-220.

V. - - Tafeln der eindeutig umkehrbaren Funktionen zweier Variabeln auf den einfachsten Zahlengebieten, Math. Ann. vol. 29 (1887) pp. 229-317.

VI. - - Ueber Algorithmen und Calculn, Arch. der Math. und Physik 2 series. vol. 5 (1887) pp. 225-278.

VII. O. Stolz and J. A. Gmeiner, Theoretische Arithmetik, 1 Abteilung, 1911.

\section{REFERENCES}

1. Harris F. MacNeish, Euler squares, Ann. of Math. (2) vol. 23 (1921-1922) pp. 221-227.

2. R. A. Fisher, The arrangement of field experiments, J. Minis. Ag. vol. 3 (1936) pp. 503513.

3. Anton Suschkewitsch, On a generalization of the associative law, Trans. Amer. Math. Soc. vol. 31 (1929) pp. 204-214.

4. C. Burstin and W. Mayer, Distributive Gruppen, J. Reine Angew. Math. vol. 160 (1929) pp. 111-130.

5. Morgan Ward, Postulates for the inverse operations in a group, Trans. Amer. Math. Soc. vol. 32 (1930) pp. 520-526.

6. M. Zorn, Theorie der alternitiven Ringe, Abhandlungen aus dem Mathematischen Seminar der Universität Hamburg (1930) pp. 123-147.

7. Anton Suschkewitsch, Über ein Elementen-system, für welche zwei Distributivgesetze gelten, Kharkovski Matematichnovo Tobarictba, series 4, VII (1934) pp. 29-32.

8. A. Adrian Albert, On a certain algebra of quantum mechanics, Ann. of Math. vol. 35 (1934) pp. 65-73.

9. David G. Rabinow, Independent sets of postulates for abelian groups and fields in terms of the inverse operations, Amer. J. Math. vol. 59 (1937) pp. 211-224.

10. B. A. Hausmann and Oystein Ore, Theory of quasigroups, Amer. J. Math. vol. 59 (1937) pp. 983-1004.

11. Herbert Boggs and G. Y. Rainich, Note on group postulates, Bull. Amer. Math. Soc. vol. 43 (1937) pp. 81-84.

12. Alfred Tarski, Ein Beitrag zur Axiomatik der Abelschen Gruppen, Fund. Math. XXX (1938) pp. 253-256.

13. Raj Chandra Bose, On the application of the properties of Galois Field to the problem of construction of hyper graeco Latin squares, Sankhya, vol. 3 pt. 4 (1938) pp. 323-338.

14. B. A. Bernstein, Postulates for abelian groups and fields in terms of non-associative operations, Trans. Amer. Math. Soc. vol. 43 (1938) pp. 1-6.

15. D. C. Murdoch, Quasigroups which satisfy certain generalized associative laws, Amer. J. Math. vol. 61 (1939) pp. 509-522.

16. H. W. Norton, The $7 \times 7$ squares, Annals of Eugenics vol. 9 (1939) pp. 269-307.

17. W. L. Stevens, The completely orthogonalized Latin squares, Annals of Eugenics vol. 9 (1939) pp. 82-93.

18. E. L. Post, Polyadic groups, Trans. Amer. Math. Soc. vol. 48.(1940) pp. 208-350.

19. G. N. Garrison, Quasigroups, Ann. of Math. vol. 41 (1940) pp. 474-487.

20. D. C. Murdoch, Note on normality in quasigroups, Bull. Amer. Math. Soc. vol. 47 (1941) pp. 134-138.

21. - Structure of abelian quasigroups, Trans. Amer. Math. Soc. vol. 49 (1941) pp. 392-409.

22. Koshichi Toyoda, On axioms of linear functions, Proc. Imp. Acad. Tokyo XVII (1941) pp. 221-227. 
23. S. Lefschetz, Algebraic topology, Amer. Math. Soc. Colloquium Publications, vol. 27, New York, 1942.

24. I. M. H. Etherinton, Non-associative arithmetics, Proceedings of the Royal Society of Edinburgh vol. 62 (1943-1944) pp. 442-453.

25. Takasaki Mituhisa, Abstractions of symmetric functions, Tôhoku Math. J. vol. 49 (1943) pp. 145-207 (in Japanese).

26. R. H. Bruck, Some results in the theory of quasigroups, Trans. Amer. Math. Soc. vol. 55 (1944) pp. 19-52.

27. G. N. Garrison, A note on invariant complexes of a quasigroup, Ann. of Math. vol. 47 (1946) pp. 50-55.

28. R. H. Bruck, Contributions to the theory of loops, Trans. Amer. Math. Soc. vol. 60 (1946) pp. 245-354.

29. Grace E. Bates, Free loops and nets and their generalizations, Amer. J. Math. vol. 69 (1947) pp. 495-550.

30. Fred Kiokemeister, A theory of normality for quasigroups, Amer. J. Math. vol. 70 (1948) pp. 99-106.

31. J. Aczel, On mean values, Bull. Amer. Math. Soc. vol. 54 (1948) pp. 392-400.

32. Grace E. Bates and Fred Kiokemeister, $A$ note on homomorphic mappings of quasigroups into multiplicative systems, Bull. Amer. Math. Soc. vol. 54 (1948)

33. A. C. Choudhury, Quasigroups and nonassociative systems. I, Bulletin of the Calcutta Mathematical Society (1948) pp. 183-194.

34. T. Evans, Homomorphisms of non-associative systems, J. London Math. Soc. vol. 24 (1949) pp. 254-260.

35. Marlow Sholander, On the existence of the inverse operations, Amer. J. Math. vol. 59 (1949) pp. 211-224.

36. B. Knaster, Sur une equivalence pour les fonctions, Colloquium Mathematicum, vol. II, Fasc. 1, 1949, pp. 1-4.

37. T. Evans, A note on the associative law, J. London Math. Soc. vol. 25 (1950) pp. 196-201.

38. Albert Sade, Quasigroups, Boulevard du Jardin-Zoologique 14, Marseilles, France, 1950.

39. Bourbaki, IV, premier partie, Les structures fondamentales de l'analyse, Livre II, Algèbre, Chapitre I, Structures algébriques, (1951).

40. G. Higman and B. H. Neumann, Groups as groupoids with one law, Publicationes Mathematicae, Tomus 2, Fasc. 3-4 1952.

41. M. Hosszu, On the functional equation of autodistributivity, Publicationes Mathematicae,

Tomas 3, Fasc. 1-2, Debrecen, 1953, pp. 83-86.

42. Paul Dubreil, Algèbre, Tome I, Paris, 1954.

43. M. Hosszu, Some functional equations related with the associative law, Publicationes Mathematicae, vol. 3, Fasc. 3-4, Debrecen, 1954, pp. 205-214.

44. Orrin Frink, Symmetric and self-distributive systems, Amer. Math. Monthly vol. 62 (1955) pp. 697-707.

45. Harry Furstenberg, The inverse operation in groups, Proc. Amer. Math. Soc. vol. 6 (1955) pp. 991-997.

46. D. A. Norton and Sherman K. Stein, An integer associated with Latin squares, Proc. Amer. Math. Soc. vol. 7 (1956) pp. 331-334.

47. - Cycles in algebraic systems, Proc. Amer. Math. Soc. vol. 7 (1956) pp. 999-1004.

48. S. K. Stein, Foundations of guasigroups, Proc. Nat. Acad. Sci. U. S. A. vol. 42 (1956) pp. 545-546.

49. M. Hosszu, On the functional equation of transitivity, Acta Sci. Math. vol. 15 (1954) pp. 203-208.

UNiversity of California, Davis, Calif. 